\title{
Signifficado, función y superación de la culpa en jóvenes universitarios: Un análisis por sexo
}

Meaning, function and overcoming of the guilt in university young people: An analysis by sex

José Luis Valdez Medina

Jorge Manzano Vivanco

Adriana Ivonne Sarandingua

Mario Ulises Maya Martínez

Mario Piña Monroy

\section{Resumen}

Norma Ivonne González Arrantia López Fuentes

El objetivo de la presente investigación consistió en conocer la concepción, manifestaciones y superación del sentimiento de culpa en jóvenes universitarios mediante una evaluación cualitativa de tipo exploratorio. Se construyó un instrumento que consta de 11 preguntas abiertas que abordan la temática de la culpa, el cual se aplicó a 300 estudiantes universitarios, repartidos equitativamente por sexo, cuyas edades oscilan entre 18 y 25 años. La aplicación se realizó a manera de entrevista de forma individual con una duración aproximada de 30 minutos por participante. Los resultados obtenidos se procesaron mediante la técnica de análisis de contenido (Alvarez-Gayou, 2005), la cual permite formular inferencias identificando de manera sistemática y objetiva características específicas de las respuestas brindadas. La investigación demuestra que tanto hombres como mujeres conciben la culpa como el sentimiento que surge después de realizar algo malo o que se cree es indebido de acuerdo con los juicios de valor, lo cual provoca remordimiento y arrepentimiento. Las estrategias que hombres y mujeres emplean para solucionar este sentimiento son la reflexión y la reparación del daño cometido al otro, sin embargo, también cabe decir que los hombres son más propensos a evadir o justificar la situación, mientras las mujeres buscan directamente la resolución. La culpa es, por tanto, una estrategia psicosociocultural que funciona como mecanismo de control social, cuyo fin consiste en regular de forma efectiva el comportamiento social del individuo, mismo que lo lleva a pedir perdón, implicando con ello el intento por recuperar la confianza propia y del otro, con el objetivo de reducir la amenaza y vivir sin miedo.

\section{Abstract}

The objective of this research was to study the definition, demonstrations and overcoming guilt in college students through exploratory qualitative assessment. An instrument was constructed consisting of 11 questions on the theme of guilt, which was applied to 300 university students, divided equally by gender, aged between 18 and 25. The application was made as an individual interview lasting approximately 30 minutes per participant. The results were processed using content analysis technique (Alvarez-Gayou, 2005), which allows to make findings identifying systematic and objective characteristics of the answers given. Research shows that men and women conceive guilt as the feeling that comes after doing something wrong or is believed to be improper according to the value judgments, which causes remorse and repentance. The strategies that men and women used to overcome this feeling are the reflection and repair damage done to the other. However, men also said that they evade or justify the situation, while women seek resolution directly. Guilt is a psycho-social-cultural strategy that serves as a mechanism of social control, its purpose is to effectively regulate the social behavior of the individual, the same that leads to apologize, implying an attempt to regain the trust itself and with the other people, in order to reduce the threat and live without fear.

Palabras Clave: Control social, construcción psicosociocultural, remordimiento, arrepentimiento, juicios de valor.

Keywords: Social control, psycho-socio-cultural construction, remorse, repentance, value judgment. 
Prácticamente, todos los seres humanos han experimentado la culpa en algún momento de su vida, pues se ha escrito que la culpa surge cuando se realizan acciones repudiadas por los demás y hasta por uno mismo (Urtubia, 2006). De acuerdo con el Diccionario Enciclopédico Barsa (1970), la palabra culpa se define como un sentimiento de responsabilidad o remordimiento por alguna ofensa, crimen o equivocación, ya sea este real o imaginario. Sin embargo, su definición varía de acuerdo con la perspectiva en que se valore.

En particular, desde la religión cristiana, los teólogos muestran que el sentimiento de culpa yace en el pecado original, tal como lo indican las Sagradas Escrituras. La falta cometida por Adán y Eva condenó a todos los hombres a vivir en el pecado, generando un sentimiento de culpabilidad producto de la falla cometida que, sin embargo, puede llegar a ser superado con un alto grado de fe, de imitación de Dios, y arrepentimiento de los pecados (Grimberg, 1988).

Por otro lado, dentro del terreno de la moral, se explica que dicho sentimiento se establece a partir de la distinción entre el bien y el mal; proceso que comienza desde la infancia, ya que, frecuentemente se educa a los niños con base en cargas afectivas que van llenas y producen culpa (Urtubia, 2006). Por ello, González (2006), comenta que cada persona dirige su vida con apoyo del llamado Código Moral; ese código es individual, aunque es el resultado de las reglas dadas por la cultura y por la familia que se van transmitiendo de generación en generación. Tales reglas y normas de vida se entrelazan, puesto que la familia es la principal institución donde se señalan para hombres y mujeres los diferentes atributos, características y funciones a desempeñar dentro y fuera de la sociedad (Valdez-Medina, 2009).

La esencia de la culpa, según Branden (2000), radica en el remordimiento de la conciencia moral, ya que la culpa contiene la implicación de elección y responsabilidad seamos o no conscientes de ello. Para la psicología, la culpa ha sido un tema fundamental; el psicoanálisis fue una de las primeras corrientes preocupadas por su estudio. Al respecto, Freud (1929), propuso dos orígenes del sentimiento de culpa: la angustia frente a la autoridad y, más tarde, la angustia frente al superyó. La primera, surge como resultado de la angustia frente a la agresión de la autoridad externa, mientras que la segunda se refiere a la instauración de una autoridad interna en cuyo caso hay igualación entre la mala acción y el propósito malo; de ahí la conciencia de culpa y la necesidad de castigo. La culpa es por definición un estado o situación subjetiva, cuyo estudio comprende la relación psíquica interna entre el superyó y el yo, que sería el responsable directo de todo sentimiento de culpa (Freud, 1953; en SpezialeBagliacca, 2002).

Para Zimbardo (1984), la culpa es un constructo que se enseña desde la cuna, pues en los primeros años del niño, todos los esfuerzos realizados para lograr su educación están dirigidos a condicionar sus conductas. Así, si éstas son inadecuadas frente a la sociedad se le reprenderá. De esta manera, el sentimiento de culpa experimentado cuando ocurre una mala conducta, previene precisamente la mala conducta sobre todo en ausencia de las figuras de autoridad que controlan las recompensas y castigos. Por lo tanto, para este autor, la vergüenza y la culpa representan los patrones paternos interiorizados.

De acuerdo con las posturas antes mencionadas, la culpa tiene su génesis en el miedo al castigo y la venganza; con lo cual se suscitan en el individuo efectos y respuestas para encontrar una solución a este sentimiento, la cual se puede dar a través del arrepentimiento y el perdón. Hecho que avala Valdez-Medina (2009), al mencionar que la culpa surge en una dinámica de evitar el castigo o la venganza y conseguir el perdón y la confianza del otro, situación que llevaría al individuo a un estado de paz o equilibrio. 
En dicho sentido, para Mc Cullough, Worthington y Rachal (1997), el perdón se concibe como una transformación motivacional en dos componentes: evitar a la persona que ha ofendido y la motivación a buscar venganza, señalando que el perdón implicaría una disminución de ambas motivaciones. Klein (1960; citada en Grimberg, 1988), hace referencia a la necesidad de anular o reparar el daño hecho al objeto amado; puesto que como refiere Freud (1929), cuando un individuo presenta dicho sentimiento y no lo soluciona, tiende a actuar de forma masoquista y muestra conductas autopunitivas o de compulsión al auto-castigo, que pueden llevar al suicidio (pulsión de muerte). Como lo confirma Custer (1979), al afirmar que el sentimiento de culpabilidad es la causa más común de desórdenes mentales y nerviosos, lo cual se manifiesta en el mal funcionamiento de diferentes órganos del cuerpo.

En concordancia con lo ya mencionado, se observa que la culpa tiene la función de control social, ya que controla la conducta de los individuos para tratar de evitar el daño a los otros, y el castigo que se puede recibir por ello. A partir de esto, puede decirse a manera de hipótesis que la culpa es un evento netamente psicosociocultural, que parte del temor a recibir un castigo por el acto cometido, y cuyo fin primordial es tratar de regular de forma efectiva el comportamiento del individuo, para recobrar la confianza perdida y volver a adaptarse a una nueva situación de paz o equilibrio (Valdez-Medina, 2009).

De esta forma, pese a que existen investigaciones acerca del tema, sobre todo en el terreno de lo jurídico, aún no se describen científicamente las diferencias que existen entre sexos sobre la concepción y resolución del sentimiento de culpa, es decir, se sabe poco acerca de las formas en que los hombres y mujeres responden ante una situación similar. Por lo tanto, el objetivo de ésta investigación es comparar las diferencias entre jóvenes universitarios de ambos sexos en torno a la concepción, mani- festaciones y resolución del sentimiento de culpa.

\section{Método}

\section{Participantes}

Se trabajó con una muestra de 300 sujetos (150 hombres y 150 mujeres), todos ellos alumnos de la Universidad Autónoma del Estado de México (UAEM), con un rango de edad de entre 18 a 25 años. La dinámica consistió en preguntar si el individuo alguna vez había sentido culpa; de contestar afirmativamente, se continuaba con la aplicación del instrumento diseñado para la investigación.

\section{Instrumento}

Se construyó un cuestionario que consta de 11 preguntas abiertas, las cuales permiten evaluar el significado, la función y la forma de solución de la culpa que experimentan los jóvenes universitarios. Las preguntas fueron: ¿qué es la culpa?, ¿ante qué situaciones surge la culpa?, ¿por qué tienen culpa las personas?, ¿para qué tienen culpa las personas?, ¿qué consiguen las personas sintiendo culpa?, ¿qué pierden las personas sintiendo culpa?, ¿qué se evita cuando se siente culpa?, ¿cómo resuelven la culpa las personas?, ¿cómo podemos detectar que una persona siente culpa?, ¿qué consecuencias genera la culpa en las personas?, ¿cómo lo resolviste?

\section{Procedimiento}

Una vez obtenida la autorización de los individuos, se realizó la aplicación del instrumento a manera de entrevista a cada participante, con una duración aproximada de 30 minutos.

\section{Análisis de datos}

Los resultados se analizaron cualitativamente con base en la técnica de análisis de 
contenido (Alvarez-Gayou, 2005), la cual permite formular inferencias identificando de manera sistemática y objetiva ciertas características específicas de las respuestas brindadas.

\section{Resultados}

El establecimiento de las categorías de resultados se realizó con base en un análisis por jueces, con un acuerdo entre ellos mayor o igual al $75 \%$. Hombres y mujeres concuerdan al referir que la culpa se traduce en remordimiento generado después de haber cometido un acto indebido.

De acuerdo con los resultados obtenidos en la pregunta ¿qué es la culpa?, ellas consideran una emoción que produce una sensación de incomodidad hacia uno mismo (en forma de castigo o auto-reprimenda) o hacia otra persona, mientras que para los hombres es vista como un sentimiento que puede traducirse de muchas maneras: ansiedad, inseguridad, rechazo (ver tabla 1).

Tabla 1.

¿Qué es la culpa?

\begin{tabular}{llll}
\hline \multicolumn{1}{c}{ Hombres } & & \multicolumn{1}{c}{ Mujeres } \\
\hline Sentimiento & 34 & Sentirse mal & 34 \\
Remordimiento & 27 & Realizar algo malo & 26 \\
Realizar algo malo & 26 & Remordimiento & 15 \\
Sentirse mal & 13 & Reacciones físicas y psicológicas & 11 \\
Sensación & 10 & Arrepentimiento & 9 \\
Control social & 10 & Conciencia & 9 \\
Arrepentimiento & 9 & Daño & 7 \\
Responsabilidad de hacer o no algo & 7 & Fuera de los valores & 7 \\
Consecuencias de un acto erróneo & 7 & Estado de ánimo & 7 \\
Daño & 4 & Malestar & 7 \\
Miedo & 3 & Conducta & 6 \\
& & Problemas & 6 \\
& & Responsabilidad de hacer o no hacer algo & 3 \\
& & Muerte & 1 \\
\end{tabular}

Con respecto a los resultados obtenidos a la pregunta ¿ante qué situaciones surge la culpa?, ambos sexos coinciden en que la culpa surge a partir de realizar actos que dañan a terceros y generan su vez arrepentimiento y remordimiento.

Sin embargo, existen diferencias en cuanto a las situaciones donde se genera. Según los hombres cualquier acto que involucre un con- flicto con otra persona o cualquier situación que atente contra las ideologías propias podría dar origen a experimentar el sentimiento. En cambio, para el sexo femenino la culpa surge cuando se actúa de forma impulsiva sin meditar antes la situación e inclusive cuando se cometen actos que atentan contra la moral de las personas generan una imagen negativa para los demás (ver tabla 2). 
Tabla 2.

¿Ante qué situaciones surge la culpa?

\begin{tabular}{|c|c|c|c|}
\hline \multicolumn{2}{|l|}{ Hombres } & \multicolumn{2}{|l|}{ Mujeres } \\
\hline Realizar acciones malas & 58 & Realizar acciones malas & 56 \\
\hline Acciones que dañan a un tercero & 22 & Situaciones que la gente cree negativas & 23 \\
\hline Problemas & 15 & Problemas & 21 \\
\hline Ideologías & 13 & Actuar sin pensar las cosas & 8 \\
\hline Situaciones emocionales & 12 & Actos inmorales & 7 \\
\hline Sentirse mal & 9 & Situaciones emocionales & 7 \\
\hline Situaciones cotidianas & 6 & Cosas malas & 6 \\
\hline Situaciones sociales & 6 & Arrepentimiento & 6 \\
\hline Respuesta pasiva & 4 & Acciones que dañan a un tercero & 4 \\
\hline Evadir responsabilidades & 3 & Mentiras & 3 \\
\hline Arrepentimiento & 1 & Evadir responsabilidades & 2 \\
\hline \multirow[t]{6}{*}{ Remordimiento } & 1 & Depresión & 2 \\
\hline & & Presión & 1 \\
\hline & & Hechos incómodos & 1 \\
\hline & & Deshonestidad & 1 \\
\hline & & Falta de comunicación & 1 \\
\hline & & Muerte & 1 \\
\hline
\end{tabular}

En las respuestas obtenidas para la interrogante ¿por qué tienen culpa las personas? Tanto hombres como mujeres aseguran que la culpa se genera por cometer un acto negativo que atenta directamente contra los valores y la moral. En contraste, existen respuestas que difieren entre ambos sexos, pues mientras que los hombres consideran la culpa como una emoción necesaria para la existencia del ser humano, las mujeres la suponen producto de las reglas sociales (ver tabla 3 ).

\section{Tabla 3.}

¿Por qué tienen culpa las personas?

\begin{tabular}{llll}
\hline \multicolumn{1}{c}{ Hombres } & & & Mujeres \\
\hline Realizar algo malo & 64 & Realizar algo malo & 54 \\
Valores-Moral & 33 & Valores-Moral & 21 \\
Por necesidad & 9 & Arrepentimiento & 16 \\
Irresponsabilidad de los actos & 7 & Actos precipitados & 13 \\
Conciencia & 6 & Tabúes sociales & 13 \\
Inseguridad & 6 & Sentirse mal & 6 \\
Sentirse mal & 5 & Dañar a alguien & 6 \\
Dañar a alguien & 5 & Irresponsabilidad de los actos & 6 \\
Consecuencias de un acto erróneo & 5 & No obtener lo deseado & 6 \\
Arrepentimiento & 3 & Consciencia & 5 \\
Actos precipitados & 2 & Remordimiento & 4 \\
Responsabilidad & 2 & & \\
Corregir un error & 2 & & \\
Remordimiento & 1 & & \\
\hline
\end{tabular}


Se observa que en la pregunta ¿para qué tienen culpa las personas? Tanto hombres como mujeres admiten que sirve para regular sus acciones. Pero al comparar entre sexos se encuentra que las mujeres presentan mayor tendencia a buscar la solución de la culpa, a di- ferencia de los hombres que lo hacen en menor grado. Ellos consideran que la culpa cumple la función de hacer sentir mal al individuo, mientras que ellas toman la situación como una razón para mejorar (ver tabla 4).

Tabla 4.

\begin{tabular}{|c|c|c|c|}
\hline \multicolumn{2}{|l|}{ Hombres } & \multicolumn{2}{|l|}{ Mujeres } \\
\hline Regular sus acciones & 32 & Corregir sus errores & 35 \\
\hline Reflexionar sobre las acciones & 26 & Sentirse mal & 23 \\
\hline Resolver los problemas & 21 & Arrepentimiento & 19 \\
\hline Remordimiento & 15 & Control & 14 \\
\hline Sentimiento & 11 & Remordimiento & 12 \\
\hline Sentirse mal & 10 & Mejorar aspectos & 11 \\
\hline Sentirse mejor & 9 & Actos indebidos & 10 \\
\hline Situaciones sociales & 6 & Sentimiento & 9 \\
\hline No repetir el mismo error & 5 & Conciencia & 7 \\
\hline Respuestas pasivas & 5 & Responsabilidad por los actos & 3 \\
\hline Actos indebidos & 4 & Sometimiento a la soledad & 3 \\
\hline Arrepentimiento & 2 & Daño & 3 \\
\hline Mejorar aspectos & 2 & Por hobbie & 1 \\
\hline Responsabilidad por los actos & 1 & & \\
\hline Justificarse & 1 & & \\
\hline
\end{tabular}

Para la cuestión ¿qué consiguen las personas sintiendo culpa?, ambos sexos coinciden en que las personas obtienen una motivación para solucionar el conflicto generador de la culpa, representando esto para el sexo masculino una forma de reencontrar el bienestar emocional, mientras que para las mujeres lo principal es la obtención de un periodo de reflexión en el cual pueden liberar los pensamientos negativos y valorar de nuevo el evento para generar un autocontrol que les permita actuar de forma distinta en situaciones futuras (ver tabla $5)$.

De acuerdo con los resultados obtenidos se encontró que para la pregunta ¿qué pierden las personas sintiendo culpa?, las mujeres argumentan que impacta directamente en la pérdida de confianza y por ende en la seguridad que cada persona tiene sobre sí misma. Los hombres, por su parte, comentan que dicho sentimiento quebranta la integridad personal. Ambos sexos coinciden en que la culpa genera una pérdida de valores que puede llegar inclusive a romper la relación con el otro, al generar en ellos un sentimiento de incomodidad e intranquilidad (ver tabla 6). 
Tabla 5.

¿Qué consiguen las personas sintiendo culpa?

\begin{tabular}{llll}
\hline \multicolumn{1}{c}{ Hombres } & & & Mujeres \\
\hline Solución de problemas & 26 & Solución de problemas & 45 \\
Bienestar emocional & 21 & Reflexionar & 22 \\
Respuestas pasivas & 20 & Sentirse mal & 15 \\
Reacciones psicológicas & 18 & Bienestar emocional & 12 \\
Reflexionar & 10 & Remordimiento & 10 \\
Responsabilizarse & 10 & Arrepentimiento & 9 \\
Tristeza & 8 & Tristeza & 9 \\
Reacciones somáticas & 7 & Sufrimiento & 7 \\
Sentirse mal & 6 & Liberar su sentimiento & 7 \\
Remordimiento & 7 & Control & 5 \\
Rechazo & 4 & Malestar & 4 \\
Liberar su sentimiento & 3 & Reacciones somáticas & 3 \\
Castigo & 3 & Problemas & 1 \\
Adaptación social & 3 & Ser responsables & 1 \\
Experiencia & 3 & & \\
Atención & 1 & & \\
\hline
\end{tabular}

Tabla 6.

¿Qué pierden las personas sintiendo culpa?

\begin{tabular}{llll}
\hline & Hombres & & Mujeres \\
\hline Respuestas pasivas & 33 & Confianza & 45 \\
Confianza & 27 & Valores & 32 \\
Valores & 19 & Tranquilidad & 31 \\
Reacciones afectivas & 16 & Relaciones afectivas & 11 \\
Tranquilidad & 15 & Motivación & 9 \\
Sentimientos negativos & 7 & Sentimiento & 6 \\
Conflicto latente & 5 & Felicidad & 3 \\
Integridad personal & 2 & Solucionar problemas & 3 \\
Felicidad & 4 & Libertad & 3 \\
Actitud & 3 & Decisión & 3 \\
Insensibilidad & 3 & Temor & 2 \\
Sufrimiento & 3 & Tiempo & 1 \\
Personalidad & 3 & Autoestima & 1 \\
Tiempo & 2 & & \\
Soluciona problemas & 2 & & \\
Autoestima & 1 & & \\
\hline
\end{tabular}


Dentro de las situaciones a evitar cuando se siente culpa ambos sexos acordaron que lo principal consiste en no repetir el acto, o bien, no verse involucrados en nuevos conflictos que puedan afectar a terceras personas. Las diferencias dejan ver que los hombres consideran la culpa como una forma de evitar responsabilizarse de los actos cometidos, mientras que las mujeres evaden sentirse rechazadas por los demás o que las traten con indiferencia (ver tabla 7).

Tabla 7.

\begin{tabular}{llll}
\multicolumn{2}{c}{ ¿Qué se evita cuando se siente culpa? } & \multicolumn{2}{c}{ Mujeres } \\
\hline Rembres & & & \\
\hline Respuesta pasiva & 35 & Repetir los mismos actos & 42 \\
Repetir los mismos actos & 26 & Problemas & 27 \\
Daños a terceros & 20 & Respuesta pasiva & 20 \\
Remordimiento & 8 & Daño a terceros & 15 \\
Estabilidad emocional & 8 & Estabilidad emocional & 14 \\
Moralidad & 8 & Actuar mal & 10 \\
Sentimiento & 8 & Sentirse mal & 5 \\
Responsabilidad & 7 & Indiferencia & 5 \\
Realidad & 6 & Culpabilidad & 3 \\
Tener contacto con el/la afectado/a & 6 & Normas sociales & 3 \\
Sentirse mal & 5 & Remordimiento & 2 \\
Solución de problemas & 4 & Realidad & 2 \\
Problemas & 3 & Reflexionar & 2 \\
Culpabilidad & 3 & & \\
Divertirse & 3 & & \\
\hline
\end{tabular}

En la interrogante ¿cómo resuelven la culpa las personas?, se encontró que hombres y mujeres creen que la mejor manera de solucionar los conflictos generadores de culpa involucra enfrentar directamente la situación, hablando, o bien, responsabilizándose de sus actos, pidiendo perdón u ofreciendo disculpas. Sin embargo, los hombres tienen una tendencia a evadir (reprimiendo, ignorando o bien deslindando) responsabilidades por la acción, en tanto, las mujeres tienden más a reflexionar y meditar la situación (ver tabla 8).

La principal manera para detectar a una persona con sentimientos de culpa se localiza en el estado de ánimo, tanto ellos como ellas argumentan que una persona con dichos sentimientos proyecta en su estado anímico la situación, a través de irritabilidad, baja autoestima y poco sentido del humor. Los hombres piensan que las personas dejan ver su culpabilidad al mostrarse nerviosos, o bien, al crear barreras con el fin de evitar hablar del tema. Las mujeres consideran que una persona muestra ese sentimiento al responsabilizarse de las acciones o al querer compensar el daño (ver tabla 9).

De acuerdo a hombres y mujeres el sentir culpa genera emociones negativas en las personas; los hombres comentan que la culpa genera estados de baja motivación, baja autoestima e inclusive puede llegar a generar reacciones somáticas como la depresión, estrés e insomnio. Para las mujeres el principal problema generado por los sentimientos de culpa se 
Tabla 8.

¿Cómo resuelven la culpa las personas?

\begin{tabular}{llll}
\hline \multicolumn{1}{c}{ Hombres } & & & Mujeres \\
\hline Resolviendo el problema & 49 & Resolviendo el problema & 48 \\
Disculpas / Perdón & 26 & Disculpas / Perdón & 14 \\
Evadir / Evitar & 14 & Actuar positivamente & 14 \\
Reflexionando & 9 & Evadir/Evitar & 12 \\
Tiempo & 9 & Responsabilidad & 11 \\
Responsabilidad & 8 & Aceptarla & 9 \\
Hablando & 7 & Hablando & 8 \\
Respuesta pasiva & 7 & Reflexionando & 8 \\
Religión & 5 & Respuesta pasiva & 6 \\
Compensando & 5 & Compensando & 5 \\
Arrepentimiento & 4 & Valores & 5 \\
Aceptarla & 4 & Madurez psicológica & 4 \\
Pedir ayuda profesional & 3 & Arrepentimiento & 3 \\
& & Justificándose & 2 \\
\hline
\end{tabular}

\section{Tabla 9.}

¿Cómo podemos detectar que una persona siente culpa?

\begin{tabular}{llll}
\hline & Hombres & & Mujeres \\
\hline Comportamiento & 35 & Sentirse mal & 32 \\
Sentirse mal & 33 & Comportamiento & 28 \\
Expresión verbal / No verbal & 24 & Expresión verbal / No verbal & 25 \\
Se crean barreras & 12 & Estado de ánimo & 19 \\
Nervios & 10 & Actitud & 14 \\
Estado de ánimo & 9 & Preocupación & 9 \\
Actitud & 9 & Respuesta pasiva & 9 \\
Preocupación & 7 & Sufrimiento & 7 \\
Respuesta pasiva & 4 & Responsabilidad & 5 \\
Sufrimiento & 3 & Arrepentimiento & 2 \\
Remordimiento & 3 & & \\
Arrepentimiento & 1 & &
\end{tabular}

traduce en malestares de diversa índole que se expresan en actitudes negativas como el enojo y la agresividad e inclusive sentimientos de soledad, desconfianza y miedo (ver tabla 10).

Para la pregunta ¿cómo lo resolviste?, hombres y mujeres consideran que la resolución del sentimiento de culpa consiste en la reflexión de la situación con el fin de buscar la manera de remediar los errores, reparar los daños o buscar compensar al otro por lo sucedido. Las mujeres van directamente a la resolución del conflicto tratando de encontrar un aprendizaje del suceso. Los hombres son más dados a evadir o justificar la situación (ver tabla 11). 


\section{Tabla 10.}

¿Qué consecuencias genera la culpa en las personas?

\begin{tabular}{llll}
\hline \multicolumn{1}{c}{ Hombres } & & & Mujeres \\
\hline Sentirse mal & 45 & Sentirse mal & 51 \\
Reacciones somáticas & 15 & Actitudes negativas & 15 \\
Inseguridad & 15 & Consecuencias psicológicas & 11 \\
Actitudes negativas & 14 & Reacciones somáticas & 11 \\
Evasión & 11 & Inseguridad & 10 \\
Preocupación & 8 & Pérdidas & 8 \\
Relaciones interpersonales & 7 & Preocupación & 8 \\
Consecuencias psicológicas & 6 & Problemas & 7 \\
Problemas & 5 & Respuesta pasiva & 6 \\
Arrepentimiento & 5 & Responsabilidad por los actos & 5 \\
Soledad & 5 & Estabilidad emocional & 5 \\
Remordimiento & 4 & Arrepentimiento & 4 \\
Pérdidas & 4 & Reflexión & 3 \\
Responsabilidad por los actos & 3 & Daños a terceros & 3 \\
Reflexión & 2 & Remordimiento & 2 \\
Suicidio & 1 & Suicidio & 1 \\
\hline
\end{tabular}

Tabla 11.

¿Cómo lo resolviste?

\begin{tabular}{llll}
\hline \multicolumn{1}{c}{ Hombres } & & & Mujeres \\
\hline Con correcciones & 26 & Con correcciones & 22 \\
Hablando & 10 & Hablando & 19 \\
Pidiendo disculpas & 9 & Aceptando el error & 11 \\
Reflexionando & 7 & Buscar la solución & 11 \\
Resolviendo & 7 & Reflexionando & 7 \\
Actuar acorde a mis principios & 7 & Pidiendo disculpas & 5 \\
Aceptando el error & 6 & Evasión & 5 \\
Asumir responsabilidades & 5 & Tiempo & 4 \\
Olvido & 4 & Asumir responsabilidades & 3 \\
Evasión & 3 & Aprendizaje & 3 \\
Arrepentimiento & 3 & Actuar acorde a mis principios & 3 \\
Enfrentando el problema & 2 & Sentirme bien & 2 \\
Cambiando mi forma de ser & 2 & & \\
Autoestima & 2 & & \\
Tiempo & 2 & & \\
Analizando el problema & 2 & & \\
\hline
\end{tabular}




\section{Discusión}

La culpa es la responsabilidad que surge ante una falta cometida. Acompaña la experiencia de hacer algo malo, herir a alguien, violar un código moral o legal (Urtubia, 2006). De acuerdo con los resultados obtenidos ambos sexos coinciden en que la culpa se traduce en remordimiento generado después de haber cometido un acto indebido. En consecuencia, es la infracción de una norma, cometida involuntariamente, o sea, sin haberla proyectado o planeado (Abbagnano, 2004). O bien, como argumenta Valdez-Medina (2009), son sólo los actos involuntarios los que generan culpa directamente, ya que los actos voluntarios podrían o no generarla.

Para sentirse culpable no sólo hay que haber transgredido una ley, pues la culpa se interioriza más allá de la falta objetiva (Caparrós \& Sanfeliú, 2004). Hombres y mujeres conciertan en que la culpa surge a partir de realizar actos que dañan a terceros o que van en contra de la integridad personal, lo que a su vez les genera arrepentimiento y remordimiento. Este resultado se denomina código moral (González, 2006), ya que, al herir a una persona se está rebasando lo permitido y al ser mal vistas socialmente estas acciones causan un desequilibrio emocional que lleva a la persona a tener un gran sentimiento de culpa.

Ellos y ellas consideran que cuando se tiene culpa se pierde autoestima, seguridad y confianza en sí mismo, lo cual perturba las relaciones afectivas. Aristegui y Bazán (2005), mencionan que al poseer una imagen débil de sí mismo, es difícil vivenciar la confianza y la amistad, así como el reconocimiento y el afecto del tejido social.

Un factor presente en ambos sexos es el considerar que la culpa lleva implícita una motivación para la solución del conflicto; dichas conductas buscan el perdón e intentan reparar el daño hecho al objeto amado (Klein, 1960 citado en Grimberg, 1988). Reafirmando la idea que señala la responsabilidad del culpable al poder decidir actuar sobre su realidad para reparar el daño, o bien, instalarse en el "no hacer" y en la indecisión, con el riesgo de "vivir para la culpa" e hipertrofiarla, ante la imposibilidad de subsanarla (Castilla del Pino, citado en Cueva Perus, 2009). Es decir, que a partir de la culpa se puede llegar a reflexionar sobre las acciones y buscar la solución del problema, hecho que se presenta con mayor frecuencia en las respuestas de las mujeres y que, de acuerdo con Valdez-Medina (2009), favorece la obtención de satisfactores y evita amenazas, situaciones que llevan a un estado de paz o equilibrio.

Una diferencia significativa entre hombres y mujeres recae en la concepción de la culpa. Para ellas, se trata de un castigo y auto-reprimenda que de acuerdo con Freud (1929), se traducirían en conductas auto-punitivas o compulsiones de auto-castigo. Mientras que para ellos es un sentimiento que se puede proyectar de diversas maneras como ansiedad, inseguridad y rechazo. Afirma Talavera (2004), que con la culpa surge la agresividad hacia sí mismo a través del auto-reproche y la crítica constante, se deja de creer en la valía personal y el sujeto se juzga como no merecedor de amor.

Este tipo de culpa, ansioso-agresiva, es más común en personas que han sido criadas en un medio ambiente con una alta censura impositiva, y se rigen por normas más estrictas sobre el comportamiento en general y de la agresión, en particular (Etxeberria, Ortíz, Conejero, \& Pascual, 2009).

De acuerdo con los resultados obtenidos, hombres y mujeres consideran que es posible reconocer cuando una persona se siente culpable, pues dicho sentimiento se expresa directamente en su estado de ánimo, generando en el individuo un malestar psicológico y emocional, que cuando no es conducido de forma adecuada, afecta su desarrollo integral y su equilibrio emocional. Talavera (2004), refiere que hay tres tipos de señales para detectar la culpa: a) físicas (presión en el pecho, dolor de estómago, de cabeza, de espalda), b) emocionales (nerviosismo, desasosiego, agresividad, irascibilidad) 
y c) mentales (pensamientos de autoacusaciones y auto-reproches).

En este sentido, los resultados encontrados muestran que hombres y mujeres consideran que los sentimientos de culpa generan reacciones psicosomáticas tales como estrés, nerviosismo y falta de sueño. Al respecto, Custer (1979), en su estudio sobre la culpa, asegura que el sentimiento de culpabilidad es la causa más común de desórdenes mentales y nerviosos y ello se manifiesta en el mal funcionamiento de diferentes órganos del cuerpo.

Por su parte, las mujeres buscan la resolución del conflicto poniendo en práctica valores como el respeto, honestidad, sinceridad y optimismo con el fin de encontrar un aprendizaje del suceso, mientras que los hombres son más dados a evadir o justificar la situación. Talavera (2004), explica que se evade la realidad para no perder la estabilidad emocional, pues si se produce un desencuentro entre nuestro ideal de cómo ha de ser nuestro comportamiento y la realidad vivida, causará dolorosos conflictos personales.

Lo anterior puede deberse a lo que plantea Urtubia (2006), al decir que los hombres que sufren de culpa son regidos por aspectos más racionales, la niegan y la colocan anteponiéndola a la sensibilidad, con una carga de autocastigo mental, que lucha contra esta sensibilidad como parte de su mundo femenino que quiere aflorar, elevando defensas, para negar y mantener que no es malo lo que se hace justificando que es hombre.

Los resultados obtenidos en la investigación son concordantes con los encontrados por Etxeberria, Ortíz, Conejero y Pascual (2009), en tanto que demuestran que son las mujeres las que muestran mayor intensidad en el sentimiento de culpa, debido a determinadas prácticas educativas que exigen más de ellas, y que todavía están en uso a pesar de creer lo contrario (Valdez-Medina, Díaz Loving, \& Pérez, 2005).
En torno a la resolución de la culpa, los participantes de esta investigación consideran que lo primero a realizarse es una reflexión de la situación o hecho acontecido, con el fin de buscar una manera de remediar los errores, reparar los daños o buscar compensar al otro por lo sucedido. Pero, en la práctica, la diferencia radica en que son las mujeres quienes van directamente a buscar solucionar el conflicto generado y con ello encontrar un aprendizaje del suceso, en comparación con los hombres, quienes son más dados a evadir el enfrentamiento del hecho o justificar su actuar.

De ahí que expertos afirmen que las prácticas educativas y una amplia gama de agentes de socialización debieran ser utilizados para reducir la tendencia a la culpabilidad ansiosoagresiva entre las mujeres y fortalecer la sensibilidad interpersonal entre los hombres (Etxeberria, Ortíz, Conejero, \& Pascual, 2009).

La mejor forma de superación del sentimiento de culpa es la resolución directa del conflicto (a través del perdón u ofreciendo disculpas a la persona dañada). Ese sería el primer gran paso para salir de la experiencia del sentimiento: el saber que se siente así porque uno mismo lo provocó. Aprender a reconocer los errores, aprender a darse cuenta de que si se está siendo el iniciador o se forma parte de un problema que genera culpa, lo ideal es reconocerlo y buscar solucionar el hecho de la mejor manera posible, y una forma de hacerlo es el perdón (Esperanza para la familia, 2010).

El perdón es el intento que realiza la persona por recuperar la confianza del otro, que equivaldría a vivir sin amenazas, ni miedos. De esta manera se regresa automáticamente a un estado de paz o equilibrio, libre de cualquier sensación de incomodidad. Pero, de no resolver la culpa se producirían efectos negativos que impactarían directamente en el estado de salud integral de las personas que la experimentan, evitando con esto la superación exitosa de la misma (Valdez-Medina, 2009). 


\section{Referencias}

Abbagnano, N. (2004). Diccionario de filosofía. México: Fondo de Cultura Económica.

Álvarez-Gayou, J.L. (2005). Cómo hacer investigación cualitativa: fundamentos y metodología. Barcelona: Paidós.

Aristegui, R. \& Bazán D. (2005). Hacia una pedagogía de la convivencia. Psykhe. Chile, 1, 137-150.

Branden, N. (2000). El respeto hacia uno mismo: como vencer el temor a la desaprobación de los demás, el sentimiento de culpa y la inseguridad. México: Paidos.

Buss, A. (1980). Self consciousness and social anxiety. San Francisco: W.H. Freeman.

Caparrós, N \& Sanfeliú, I. (2004) Orfandad, culpa y duelos. Daños colaterales. Clínica y salud, 15(3), 387-393.

Cueva Perus, M. (2009). La oscuridad en los ojos: anomia, sacrificio y culpa en las sociedades latinoamericanas. Liminar. Estudios sociales y humanísticos, 7(1), 94-111.

Custer, D. (1979). La mente en las relaciones humanas. México: Continental.

Diccionario Enciclopédico Barsa. (1970). Madrid: Barsa Planeta, Inc.

Esperanza para la familia. (2010). El sentimiento de culpabilidad. Extraído de www.esperanzaparalafamilia.com/Rev/Articulos/PDFs/ PDF0255.pdfSimilares

Etxeberria, I., Ortiz, MJ, Conejero, S. \& Pascual, A. (2009). La intensidad de la culpa habitual entre hombres y mujeres: Las diferencias en la sensibilidad interpersonal y la tendencia hacia una culpabilidad ansioso-agresiva. Revista Española de Psicología, 12 (2), 540-554.

Freud, S. (1929). Obras completas (Tomo XXI). Buenos Aires; Amorrortu.

González (2006). La culpa, con sentido. Extraído de http://www.prensa. com/actualidad/psicologia/2006/12/07index.htm)

Grimberg, L. (1988). Culpa y Depresión. Madrid; Alianza.

Mc Cullough, M. E., Worthington, E. L. \& Rachal, K. C. (1997), Interpersonal forgiving in close relationships. Journal of Personality and Social Psychology, 73, 321-336.

Speziale-Bagliacca, R. (2002). La culpa. Consideraciones sobre el remordimiento, la venganza y la responsabilidad. Madrid: Biblioteca Nueva.

Talavera, C. (2004). El sentimiento de culpa: no nos castiguemos. México: Consumer.

Urtubia, E. (2006). Creatividad en los sentimientos. Centro Psicosocial Ediciones.

Valdez Medina, J. L. (2009). Teoría de la paz o equilibrio: una nueva teoría que explica las causas del miedo y del sufrimiento, y que nos enseña a combatirlos. México: EDAMEX.

Valdez-Medina, J.L., Díaz Loving, R., \& Pérez, B.R. (2005). Los hombres y las mujeres en México: dos mundos distantes y complementarios. México: Universidad Autónoma del Estado de México.

Zimbardo, P. (1984) Psicología y vida. México: Trillas. 